\title{
Propriétés fonctionnelles - Modifications chimiques des protéines alimentaires et étude des relations structure/fonction
}

\section{Functional properties of food proteins - Chemical modifications and study of structure/function interactions in proteins}

Oléagineux, Corps Gras, Lipides. Volume 10, Numéro 1, 41-6, Janvier - Février 2003, Protéines et lipides végétaux : interactions nutritionnelles et fonctionnelles

Auteur(s) : Véronique VIDAL, Eliane CASES, Jean-Louis CUQ, Laboratoire Génie Biologique et Sciences des Aliments, Université Montpellier II, 34095 Montpellier Cedex 5, France.

Author(s) : Véronique VIDAL, Eliane CASES, Jean-Louis CUQ

Résumé : Les modifications chimiques des protéines alimentaires sont réalisées dans le but $d$ ' améliorer leurs propriétés fonctionnelles. Elles sont également pratiquées pour des études fondamentales des relations structure/fonction des protéines. Les principales modifications chimiques des protéines alimentaires sont présentées ainsi que leurs incidences sur les propriétés fonctionnelles telles les propriétés émulsifiantes, moussantes, visqueuses, d'hydratation et de gélification.

Summary : Chemical modifications are used to improve functional properties of food proteins. They are also used for fundamental studies of structure/function relationships. The main chemical modifications of food proteins are reviewed. Resulting typical functional properties including emulsification, foaming, hydration, viscosity and gelation were presented.

Mots-clés : modification chimique, protéines.

Keywords : chemical modification, proteins.

\section{ARTICLE}

Introduction

Au cours des procédés de transformation des aliments, les protéines sont soumises à des modifications liées à des traitements physiques (thermiques ou mécaniques), enzymatiques (hydrolyse, réticulation) ou chimiques (glycosylation par réaction de Maillard). Ces modifications, souvent souhaitées parfois subies, ne sont pas forcément entièrement maîtrisées. Toutefois, des modifications chimiques peuvent être réalisées de façon intentionnelle, soit pour améliorer certaines des propriétés fonctionnelles des protéines, soit pour leur en conférer d'autres. De nombreux articles ont été consacrés aux procédés de modifications des protéines en vue d' améliorer leurs propriétés fonctionnelles [1-7]. Les modifications chimiques des protéines sont également pratiquées au cours d'études plus fondamentales des relations structure fonction des protéines [8]. Actuellement, les 
Matières Protéiques Végétales font l'objet de nombreuses études leur permettant d'être mieux connues et exploitées, elles sont devenues un ingrédient important en industrie alimentaire. Parmi les voies explorées, les modifications chimiques générant des "propriétés fonctionnelles" souhaitées font l' objet de développements importants en particulier en industrie non alimentaire. Néanmoins, certaines modifications chimiques peuvent se heurter aux problèmes majeurs que sont la toxicité ou la réduction de la valeur nutritionnelle. Ces inconvénients non négligeables vont donc limiter les applications dans le domaine alimentaire aux études structure/fonction et conduire à des applications dans le domaine non alimentaire.

Les principales classes de réactions utilisées et leurs conséquences sur les propriétés fonctionnelles des protéines sont présentées dans les pages suivantes.

\section{Une grande diversité de modifications chimiques}

Les principales classes de réaction chimiques utilisées sont l' acylation, l' alkylation, la réduction, I' oxydation et la réticulation. Ces modifications chimiques des chaînes latérales des protéines, spécifiques ou non de certains résidus $d^{\prime}$ acides aminés, permettent d' obtenir des propriétés modifiées et souvent améliorées. Parmi celles ci, nous pouvons noter la solubilité, les capacités moussantes, émulsifiantes, gélifiantes, les capacités de rétention d' eau, les propriétés filmogènes.

Les chaînes latérales d' acides aminés des protéines peuvent être modifiées de diverses façons, selon le réactif employé. Néanmoins, une chaîne latérale donnée peut réagir avec plusieurs réactifs, de même qu' un réactif peut interagir avec plusieurs types de chaîne latérale (alpha- ou epsilon-aminé, hydroxyle, phénol, thiol, imidazole, indole...). Par conséquent, peu de réactifs sont spécifiques d' un type de chaîne latérale. Quant à la réactivité du groupement, elle est essentiellement fonction de I' accessibilité du groupement, et des conditions du milieu $(\mathrm{pH}$, température, sels, nature du solvant...) qui rendront spécifiques la dite réaction.

\section{Augmentation du caractère apolaire de la protéine}

De par sa facilité à mettre en ?uvre, l'acylation au moyen d' anhydrides est la méthode la plus couramment employée pour modifier les chaînes latérales des protéines. Lorsqu' elle est réalisée avec de l' anhydride acétique, la réaction conduit à l'acétylation des groupements alpha- ou epsilonaminés des protéines (figure 1a): ces groupements ionisables sont alors transformés en groupements non ionisables. La présence du groupement méthyl permet une légère augmentation $\mathrm{du}$ caractère apolaire de la protéine qui pourra alors réagir avec $\mathrm{d}^{\prime}$ autres composés apolaires de type liquide ou gazeux. Néanmoins, il apparaît que l'augmentation du caractère apolaire de la protéine est largement dépendante du taux de modification appliqué. Des études réalisées sur des protéines de légumineuses montrent que de faibles taux d'acétylation induisent une diminution de I'hydrophobicité de surface des protéines suite à l'internalisation de groupements hydrophobes au sein de la structure protéique. Des taux d'acétylation plus importants (supérieurs à $60 \%$ ) conduisent à une augmentation de l'hydrophobicité de surface des protéines. Cette augmentation serait induite par une augmentation de la charge nette négative impliquant un dépliement de la structure 
protéique et une exposition supplémentaire des groupements hydrophobes [9]. Les conséquences d'une telle modification chimique vont donc être fortement dépendantes de nombreux facteurs tels la structure primaire, la conformation de la chaîne protéique, les conditions environnementales $(\mathrm{pH}$, température, force ionique...) et le taux de réactivité. L'acétylation a été réalisée dans le but d'améliorer les propriétés émulsifiantes et moussantes de globulines constitutives des graines de légumineuses [10-13]. Parallèlement, le degré d' hydrophobicité étant accru, les interactions hydrophobes intra et intermoléculaires favorisent le plissement et l'agrégation de la protéine, abaissant ainsi ses propriétés $d^{\prime}$ absorption d' eau [14]. Pour ces mêmes raisons, les protéines acétylées présentent une sensibilité élevée à l'action de la chaleur [15].

L'augmentation du caractère apolaire d'une protéine peut également être réalisée par la mise en ?uvre de chlorures d'acides, d'esters activés d'acides gras ou bien d'anhydrides [16-20]. Ces réactions permettent le greffage de chaînes aliphatiques plus ou moins longues ou de groupements aromatiques; elles sont également appelées réactions de lipophilisation (figure $1 b$ ). Ces procédés permettent d'obtenir des protéines à caractère amphipolaire très marqué. Ces changements de structure entraînent le plus souvent une amélioration des propriétés de surface telles que les propriétés moussantes et émulsifiantes. De plus, cette amélioration semble d'autant plus importante que la longueur de la chaîne aliphatique greffée augmente. Le greffage d'acide gras a été réalisé par biocatalyse (action de lipases) sur des isolats de protéines de soja. Un rendement de greffage de $60 \%$ des résidus de lysine des isolats de protéines de soja a été atteint avec de l'acide caprique [21]. Il est à noter que le greffage n'est pas spécifique des résidus de lysine mais la liaison amide formée avec ces résidus est très stable chimiquement vis-à-vis des conditions de $\mathrm{pH}$ et de température. Les réactions de lipophilisation réalisée par biocatalyse représentent une alternative intéressante au procédé purement chimique car elles ouvrent une nouvelle voie pour la valorisation des protéines végétales en tant qu'agents tensioactifs dans le domaine des produits cosmétiques, pharmaceutiques ou d'hygiène.

$L^{\prime}$ alkylation réductrice en présence $d^{\prime}$ un aldéhyde ou d' une cétone, et $d^{\prime}$ un agent réducteur, permet également la transformation de divers groupements ionisables (aminés, indole, thiol) en groupement apolaire. La réaction présentée sur la figure $1 c$ met en évidence la réduction d' un groupement epsilon-aminé d' une protéine avec le formaldéhyde en présence de cyanoborohydrure de sodium. Le nombre de charges positives diminuant, cela se traduit par une diminution de la valeur du pHi de la protéine et donc une modification de ses propriétés de solubilité. L'alkylation réductrice à l'acétaldéhyde d'hydrolysats trypsiques de caséines induit une augmentation de la solubilité des produits de la réaction quel que soit le pH ainsi que de leur capacité émulsifiante [22].

Diminution de la charge électrique et augmentation de la polarité

L' acylation réalisée à $\mathrm{I}^{\prime}$ aide $\mathrm{d}^{\prime}$ isocyanate (figure $2 a$ ) ou carbamylation, permet le blocage des groupements epsilon-aminés et le greffage de groupement carbamyl non ionisables, aptes à former des liaisons hydrogène. Cela se traduit par une augmentation de la capacité $d^{\prime}$ absorption $d^{\prime}$ eau de la protéine et de sa solubilité. Toutefois, il est à noter que de par sa toxicité, l' isocyanate ne peut être utilisé dans le domaine alimentaire. 
L'amidation des groupements carboxyliques des protéines permet la transformation du groupement carboxylique en amine primaire ou secondaire (figure $2 b$ ). Cette réaction est utilisée pour le greffage $d^{\prime}$ acides aminés essentiels sur les protéines : en ce sens, il a pu être greffé de la méthionine sur des protéines de soja ou du tryptophane sur les protéines de gluten de blé [23]. De même, du tryptophane, de la glycine, de l'alanine, de la méthionine, de l'acide aspartique et de l'asparagine ont été incorporés sur des caséines par formation d'une liaison isopeptidique [24]. Cette liaison isopeptidique est une liaison amide entre le groupement carboxylique de l'acide aminé à greffer et le groupement epsilon-aminé des résidus de lysine d'une protéine ou bien le groupement aminé de l'acide aminé et un groupement carboxylique libre de la protéine. Les liaisons isopeptidiques formées étant hydrolysables pour la plupart par une aminopeptidase digestive, la réaction d' amidation $\mathrm{n}^{\prime}$ altère pas la valeur nutritionnelle de la protéine modifiée. La réaction $\mathrm{d}^{\prime}$ amidation permet également la protection des groupements epsilon-aminés contre la réaction de Maillard.

La réaction $d^{\prime}$ alkylation réductrice réalisée à partir $d^{\prime}$ un intermédiaire de la réaction de Maillard (la base de Schiff) et en présence de borohydrure, permet la formation de néoglycoprotéines (figure 3). La présence de ces résidus glycosylés rend la protéine modifiée plus polaire, ses propriétés de solubilité étant alors accrues [25]. Les néoglycoprotéines ainsi formées présenteraient des propriétés interfaciales améliorées. L'alkylation réductrice réalisée sur des caséinates ou des protéines de pois a permis de greffer différents carbohydrates (glucose, fructose, lactose ou galactose) : les dérivés néoglycosylés qui en résultent présentent de meilleures propriétés émulsifiantes que les protéines natives $[26,27]$.

Accroissement de la charge négative de la protéine (par introduction de groupements ionogènes)

L' accroissement de la charge négative d' une protéine peut être réalisé par greffage de groupements carboxyles ionisables. La présence de charges négatives additionnelles entraîne une diminution du pHi de la protéine, une augmentation des répulsions électrostatiques, le déplissement de la protéine et sa dissociation. Elle se traduit par une amélioration de sa dispersibilité, sa capacité d' absorption $d^{\prime}$ eau et sa sensibilité à la précipitation par les ions bivalents type calcium [28-33]. L' introduction de groupes carboxyles ionisables peut être réalisée par acylation avec lanhydride succinique (succinylation), carboxyméthylation avec l]acide iodoacétique ou par désamidation des résidus de glutamine et d'asparagine provoquée dans des conditions de températures élevées.

L' acylation au moyen d' anhydride succinique ou succinylation (figure 4a) améliore les propriétés gélifiantes des protéines de poisson [34], mais altère l' aptitude des micelles de caséine du lait à coaguler par voie enzymatique et acide. En effet, pour un taux de succinylation des résidus de lysine de l'ordre de $20 \%$, les charges négatives additionnelles induisent des répulsions entre protéines qui modifient l'intégrité micellaire et diminuent les propriétés de coagulation des caséines [35-37]. Parallèlement, une amélioration des propriétés émulsifiantes et moussantes de la caséine a été observée [23] lorsque plus de $40 \%$ de ses groupes epsilon-aminés sont succinylés. Le remplacement des forces électrostatiques de type attractif par des forces répulsives entre les groupements 
carboxyliques additionnels et les charges négatives proximales provoque une diminution des interactions inter et intra-protéiques au profit des interactions protéines-eau. La succinylation des protéines de soja améliore leur solubilité à un $\mathrm{pH}$ donné. Le déploiement des chaînes polypeptidiques favorise ainsi I' adsorption interfaciale et la formation d' un film interfacial. [38]. Réalisée sur des isolats de protéines de tournesol, elle induit un abaissement du point isoélectrique (déplacement du minimum de solubilité vers des valeurs de $\mathrm{pH}$ acides) et une diminution de leur capacité émulsifiante [30].

La carboxyméthylation (figure 4b) à l'aide d'haloacétates (ex: acide iodoacétique) ou d'haloalkylamides (ex : iodoacétamide) est également utilisée pour accroître la charge négative de la protéine. La réaction peut avoir lieu sur les groupements epsilon-aminés, phénol, imidazole, indole ou thiol et thioéther des protéines.

Réticulation Formation de ponts covalents

La réticulation des protéines a pour conséquence un abaissement de la solubilité de la protéine et de sa digestibilité. Elle peut se faire par la formation de ponts covalents disulfure qui est induite par oxydation modérée des groupements thiol en présence $d^{\prime}$ air, de bromate ou de peroxyde $d^{\prime}$ hydrogène dans certaines conditions (figure $5 a$ ). Cette réaction est utilisée en technologie boulangère pour améliorer les propriétés viscoélastiques du gluten de blé.

La rupture des ponts disulfure peut être obtenue par action d'agents réducteurs du type mercaptoéthanol, dithiothréithol, cystéine ou encore des sulfites, ces derniers permettant une réversibilité de la réaction (figure $5 b$ ). Le bisulfite de sodium présente une meilleure efficacité sur les protéines de soja que le sulfite de sodium ou la cystéine [39]. Elle se traduit par une augmentation de I'indice de dispersibilité des protéines de soja relatif à la dissociation majoritaire des globulines $7 \mathrm{~S}$ et $11 \mathrm{~S}$ liées entre elles par des liaisons disulfure covalentes. Le passage dans des conditions de $\mathrm{pH}$ alcalin permet également d'augmenter ce taux de dissociation. Ces réactions sont souvent utilisées pour améliorer la solubilité de protéines et faciliter leur purification. Ces additifs sont d'ailleurs fréquemment utilisés dans la technologie de fabrication des films protéiques à base de protéines de gluten de blé. La rupture des ponts disulfure intermoléculaires nécessaire à la solubilisation préalable des globulines requiert l'utilisation de sulfites. Les S-sulfonates formés redonnent des groupements thiols au cours de la phase de séchage du film. L'oxydation de ces groupements permet l'établissement de nouveaux ponts disulfure, peu abondants mais d'énergie élevée, qui confèrent au film sa rigidité.

La réticulation entre deux groupements epsilon-aminés de la lysine peut également avoir lieu via l' utilisation d'agents bifonctionnels tels que les aldéhydes malonique, glutarique ou le formaldéhyde [40-41]. Ces agents sont également utilisés pour améliorer les propriétés filmogènes des protéines d'origine végétales, en particulier pour améliorer la résistance mécanique de films protéiques à base de protéines de gluten de blé [42] ou de protéines de coton [43]. L'étude du phénomène de réticulation par le formaldéhyde, le glutaraldéhyde et le glyoxal pendant la formation 
du film protéique a permis de montrer que les acides aminés majoritairement impliqués étaient la lysine (en présence de dialdéhydes), la tyrosine (en présence de formaldéhyde) et l'arginine (en présence de glyoxal). Le gossypol, pigment phénolique présent naturellement dans des glandes de certaines espèces de cotonniers a également été employé comme agent réticulant pour la fabrication de film à base de protéines de coton. Le gossypol interagit avec les résidus de lysine au niveau de sa fonction aldéhydique avec formation d'un dérivé quinonimique. Le traitement des protéines de coton par le gossypol permet d'augmenter la force à la rupture et diminuer la solubilité des films. En outre, ces matériaux présentent des propriétés de biodégradabilité intéressantes qui permettent d'envisager des applications pour la fabrication d'emballages biodégradables [44]. Néanmoins, une alternative à ce type de modifications par voie chimique est l'utilisation d'enzymes "pontantes" telle la transglutaminase [45].

\section{CONCLUSION}

De toute évidence, la liste des modifications chimiques applicables aux protéines est très importante. $L^{\prime}$ ensemble des réactions décrites dans cet article n' est pas exhaustive mais démontre simplement la diversité des possibilités de modifications de la nature des protéines en vue de modifier leurs propriétés fonctionnelles. Néanmoins, les conséquences nutritionnelles et toxicologiques peuvent représenter un frein non négligeable dans I' utilisation de certaines de ces modifications en industrie agroalimentaire. Aujourd'hui d'autres types d'applications sont développées dans le domaine de l'analyse spectrale par exemple [40]. L'avenir de ces méthodes est très prometteur si l'on considère leur utilisation à des fins non alimentaires ou dans le cadre d'étude des relations structure/fonction.

\section{REFERENCES}

Feeney RE. Chemical modification of food proteins. In Food proteins: Improvement through chemical and enzymatic modification, RE Feeney, JR Whitaker, ed. Washington: American Chemical Society ; $1977: 3-36$

Kinsella JE, Shetty KJ. Chemical modification for improving functional properties of plant and yeast proteins. In: Functional and protein structure, A Pour El, ed. Washington: American Chemical Society ; 1979 : 37-63.

Kinsella JE, Whitehead, DW. Modification in milk proteins to improve functional properties and applications. In: Milk - The vital force, D Reidel Publishing ; 1987 : 791-804.

Kester JJ, Richardson T. Modification of whey proteins to improve functionality. J Dairy Sci 1984 ; 67 : 2757-74. 
Richardson T. Chemical modifications and genetic engineering of food proteins. J Dairy Sci $1985 ; 68$ : 2753-62.

Gruener L, Ismond MAH. Effect of acetylation and succinylation on the physicochemical properties of the canola 12 S globulin. Part I. Food Chem 1997 ; 60 : 357-63.

Lakkis J, Villota R. Effect of acylation on substructural properties of proteins: A study using fluorescence and circular dichroism. J Agric Food Chem $1992 ; 40$ : 553-60.

Cayot $\mathrm{P}$, Courthaudon JL, Lorient D. Emulsifying properties of pure and mixed alphas ${ }_{1}$ - and betacasein fractions: effects of chemical glycosylation. J Agric Food Chem $1991 ; 39$ : 1369-73.

Schwenke KD, Dudek S, Mothes R, Raab B, Seifert A. Physicochemical and enzymatic studies on acetylated protein isolates from faba beans (Vicia faba L.). Nahrung 1993 ; 37 : 258-68.

Kim KS, Rhee JS. Effect of acetylation on emulsifying properties of glycinin. J Agric Food Chem $1990 ; 38: 669-74$.

Schmandke H, Schultz M, Schmidt G, Schneider C, Andersson O. Tension of oil-water interface and properties of O W emulsions in dependence of faba bean globulins. Nahrung $1990 ; 34: 363-8$.

Gruener L, Ismond MA. Effets of acetylation and succinylation on the functional properties of the canola $12 S$ globulin. Food Chem $1997 ; 60: 513-20$.

Husband FA, Wilde PJ, Clark DC, Rawel HM, Muschiolik G. Foaming properties of modified faba bean protein isolates. Food Hydrocoll $1994 ; 8: 455-68$.

Kinsella JE, Whitehead DM, Brady J, Bringe NA. Milk proteins: possible relationhips of structure and function. In: Developments in Dairy Chemistry-4. PF Fox, ed. London: Elsevier Applied science 1989 ; $55-95$

Klepack AM, Porzucek $\mathrm{H}$, Kluczynska M. Effect of heat treatment on chemically modified proteins of legume seeds. Food chem $1997 ; 58: 219-22$.

Roussel-Philippe C, Pina M, Graille J. Chemical lipophilization of soy protein isolates and wheat gluten. European Journal of Lipid Science and Technology 2000 ; 102 : 97-101. 
Haque Z, Kito M. Lipophilization of soybean glycinin: covalent attachment to long chain fatty acid. Agric Biol Chem 1982 ;46 : 597-9.

Haque Z, Kito M One step lipophilization of proteins using fatty acyl anhydride. Agric Biol Chem $1984 ; 48$ : 1099-2001.

Haque Z, Kito M Lipophilization of alphas ${ }_{1}$-casein. 3. Purification and physicochemical properties of novel amphipathic fatty acyl peptides. J Agric Food Chem $1984 ; 32$ : 1392-7.

Akita EM, Nakai S Lipophilization of beta-lactogloguline: Effect on hydrophobicity and surface functional properties. J Food Sci $1990 ; 55: 711-7$.

Roussel C, Pina M, Graille J, Huc A, Perrier E Lipophilisation des protéines biocatalysées par des lipases ; cas des isolats de protéines de soja. Ol Corps Gras Lipides 1997 ; 4 : 284-9.

Touati A, Chobert JM, Bertrand-Harb C, Dalgalarrondo M, Nicolas MG, Haertlé T. Reductive alkylation of -casein tryptic peptides. Solubility and emulsifying properties. Milchwissenschaft $1990 ; 45: 416-$ 20.

Kinsella JE, Shettykj. Chemical modification for improving functional properties of plant and yeast proteins. In Functional and Protein Structure. A Pour El, ed. Washington: American Chemical Society ; $1979:$ 37-63.

Puigserver AJ, Gaertner HF, Sen LC, Feeney RE, Whitaker JR. Covalent attachment of essential amino acids to proteins by chemical methods. Nutritional and functional significance. In Modification of proteins: Food, nutritional, and pharmacological aspects. RE Feeney, JR Whitaker, ed. Washington: American Chemical Society ; $1982: 149-68$.

Kitabatake N, Cuq JL, Cheftel JC. Covalent binding of glycosyl residues to beta-lactoglobulin: effect on solubility and heat stability. J Agric Food Chem $1985 ; 33: 125-30$.

Canton MC, Mulvihill DM. Functional properties of caseinates chemically modified by reductive alkylation of lysine with reducing sugars. In Proc Int Dairy Fed Symp Physico-chemical aspects of protein-rich milk products. Denmark ; 1983 : 339-42.

Pedrosa C, Trisciuzzi C, Ferreira S. Effects of glycosylation on functional properties of vicilin, the 7S storage globulin from pea (Pisum Sativum). J Agric Food Chem 1997 ; 45 : 2025-30. 
Kim SH, Kinsella JE. Surface active properties of proteins: Effects of progressive succinylation on film properties and foam stability of glycinin. J Food Sci $1987 ; 52: 1341-5$.

Schwenke KD, Rauschal EJ. Effect of succinylation on the physico-chemical properties of some food proteins. Nahrung $1980 ; 24: 593-5$.

Schwenke KD, Rauschal EJ, Zirwer D, Linow RJ. Structural changes of the $11 \mathrm{~S}$ globulins from sunflower (Helianthus annus L.) after succinylation. Int J Peptid Prot Res 1986 ; 25 : 347-54.

Schwenke KD, Zirwer D, Gast K et al.. Dissociation of $11 \mathrm{~S}$ globulins from plant seeds after succinylation a problem of subunit interaction. In Protein interactions. H Visser, ed. VCH: Weinheim ; 1992 : 233-52.

Schwenke KD. Enzyme and chemical modification of proteins. In Food proteins and their applications, S Damodaran, A Paraf, ed. New York: Marcel Dekker ; 1997 : 393-423.

Rao AGA, Rao MSN. Effect of succinylation on the oligomeric structure of glycinin.. Int J Peptid Prot Re $1979 ; 14$ : 307-12.

Chen LF, Richardson T, Amundson CH. Some functional properties of succinylated proteins from fish protein concentrate. J Milk Food Tech 1975 ; 38 : 89-93.

Vidal V, Gastaldi E, Lefebvre E et al. Effect of succinylation on the rennet coagulation of milk. J Dairy Sc $1998 ; 81: 69-75$.

Vidal V, Marchesseau S, Lagaude A, Cuq JL. Influence of chemical agents on casein inte ractions in dairy products: chemical modification of milk proteins. Colloids and Surfaces B: Biointerfaces $1998 ; 12$ : 7-14.

Vidal V, Marchesseau S, Cuq JL. Physicochemical Properties of Acylated Casein Micelles in Milk. J Food Sci $2002 ; 67: 42-7$.

Franzen KL, Kinsella E. Functional properties of succinylated and acetylated soy protein. J Agric Food Chem $1976 ; 24: 788-95$. 
Abtahi S, Aminlari M. Effect of sodium sulfite, sodium bisulfite, cysteine, and pH on protein solubility and sodium dodecyl sulfate-polyacrylamide gel electrophoresis of soybean milk base. J Agric Food Chem $1997 ; 45: 4768-72$.

Means GE, Feeney RE. Chemical modifications of proteins: A review. J Food Biochem 1998 ; 22 : 399426.

Gerrard JA, Brown PK, Fayle SE. Maillard crosslinking of food proteins. II: the reactions of glutaraldehyde, formaldehyde and glyceraldehyde with wheat proteins in vitro and in situ. Food chem $2003 ; 80: 35-43$.

Guilbert S, Cuq B, Gontard N. Recent innovations in edible and/or biodegradable packaging materials. Food Addit Contam $1997 ; 14$ : 741-51.

Marquie C. Chemical reactions in cottonseed protein cross-linking by formaldehyde, glutaraldehyde, and glyoxal for the formation of protein films with enhanced mechanical properties. J Agric Food Chem $2001 ; 49$ : 4676-81.

Marquie C, Aymard C, Cuq JL, Guilbert S. Biodegradable packaging made from cottonseed flour: formation and improvement by chemical treatments with gossypol, formaldehyde, and glutaraldehyde. J Agric Food Chem $1995 ; 43$ : 2762-7.

De Jong GAH, Koppelman SJ. Transglutaminase catalyzed reactions: Impact on food applications. J Food Sci $2002 ; 67: 2798-806$. 


\section{Illustrations}

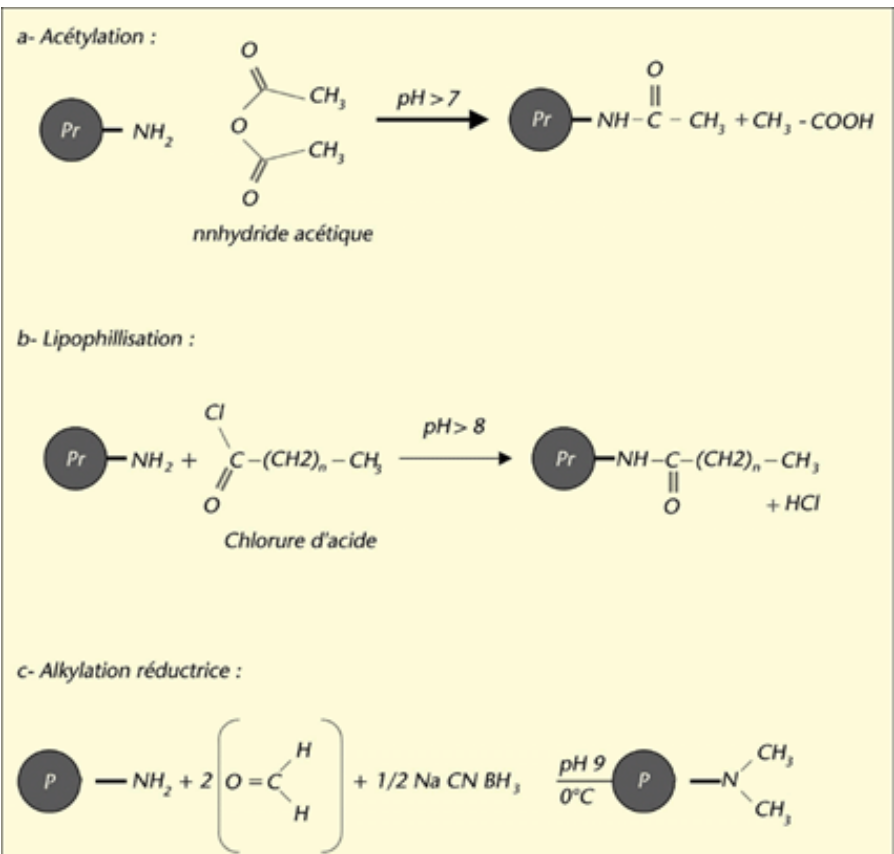

Figure 1 - Schéma réactionnel de modifications chimiques induisant une augmentation du caractère apolaire des protéines.

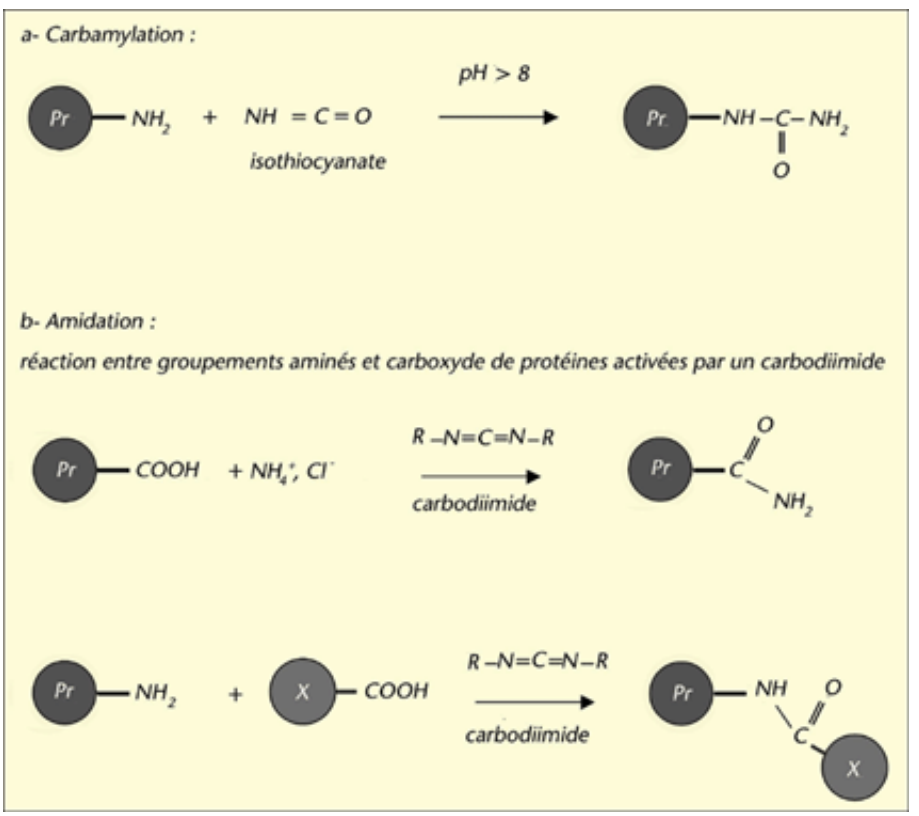

Figure 2 - Schéma réactionnel de modifications chimiques induisant une diminution de la charge électrique et une augmentation de la polarité des protéines. 


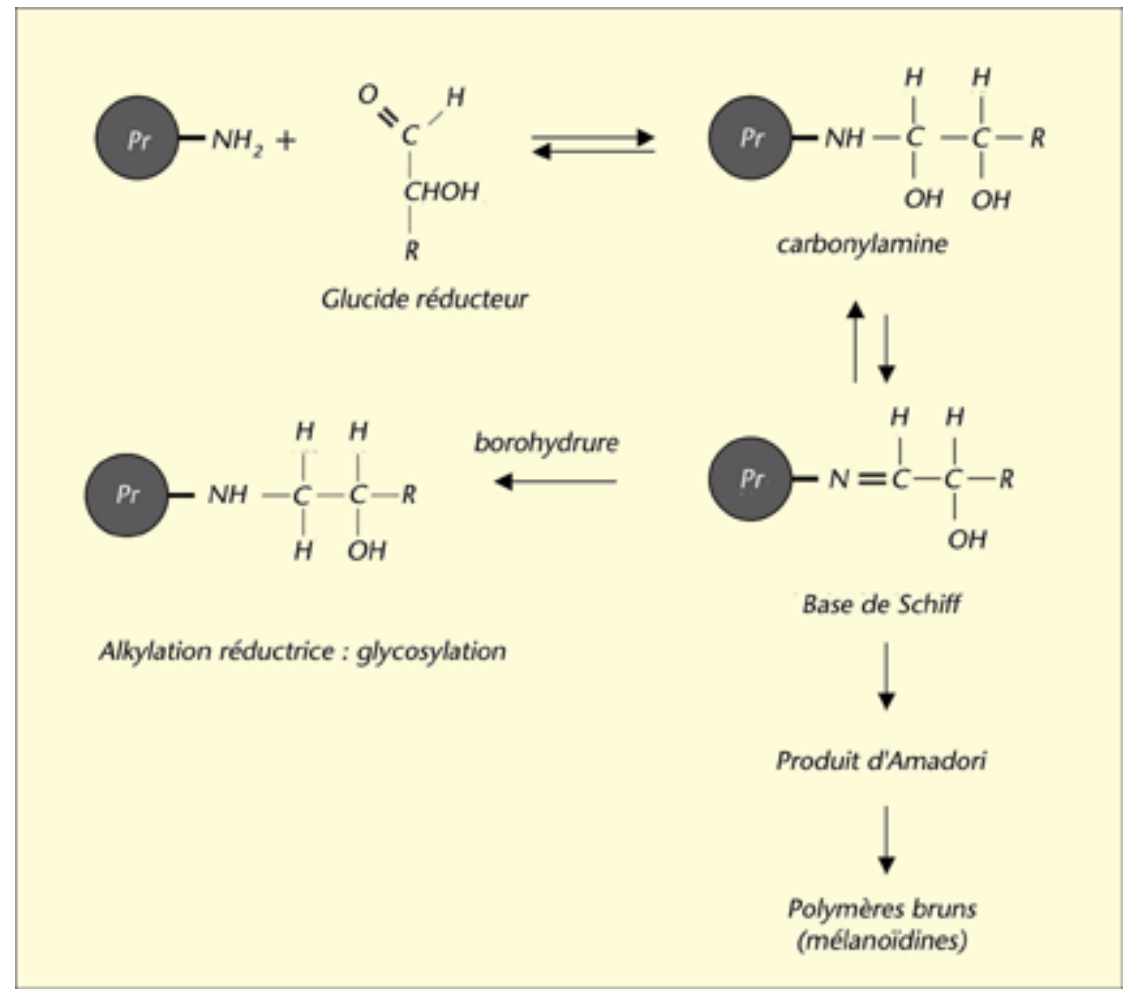

Figure 3 - Schéma réactionnel de l'alkylation réductrice réalisée à partir d' un intermédiaire de la réaction de Maillard : formation de néoglycoprotéines.

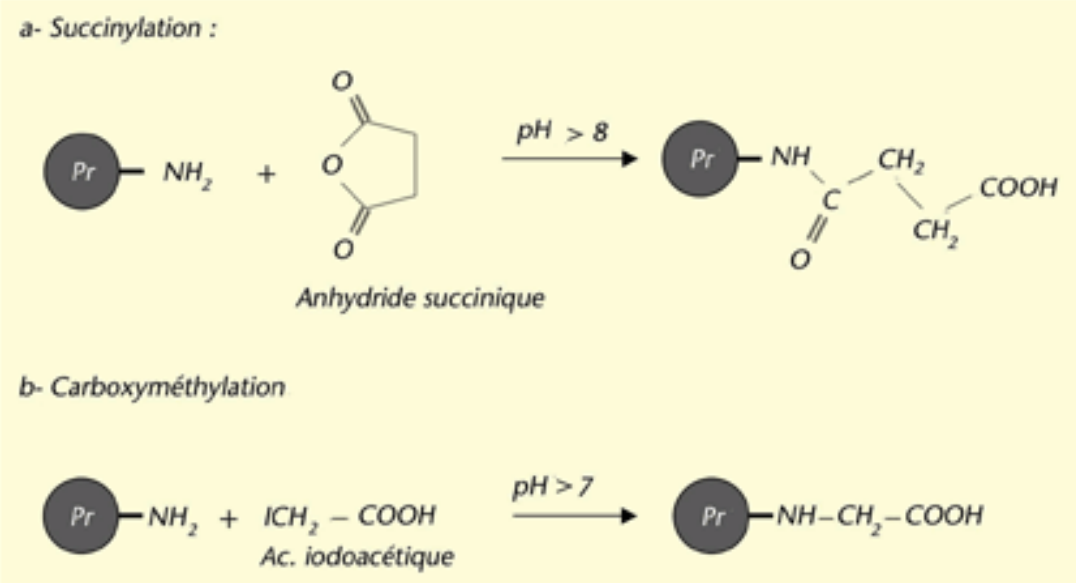

Figure 4 - Schéma réactionnel de modifications chimiques conduisant à l'introduction d'un groupement carboxylique. 
a- Oxydation :

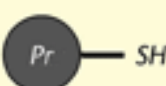

air, bromate, $\mathrm{H}_{2} \mathrm{O}$

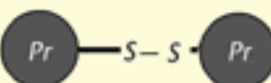

b- Réduction, Sulfitolyse :

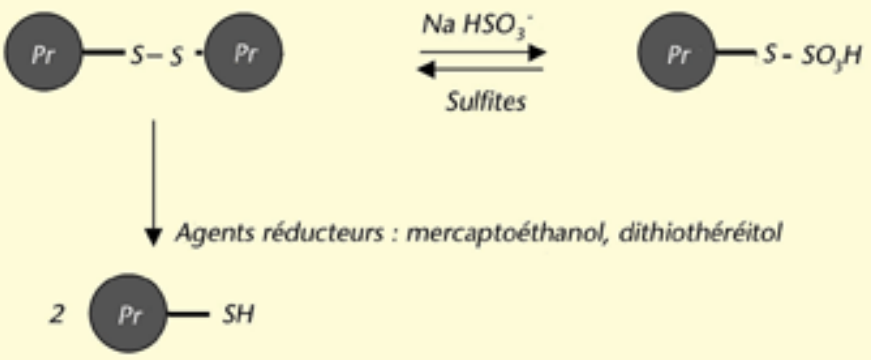

Figure 5 - Schéma réactionnel de modifications chimiques induisant la formation ou la rupture de ponts covalents entre acides aminés soufrés 